\title{
Mapping and signaling of neural pathways involved in the regulation of hydromineral homeostasis
}

\author{
J. Antunes-Rodrigues ${ }^{1}$, S.G. Ruginsk ${ }^{1}$, A.S. Mecawi ${ }^{1}$, L.O. Margatho ${ }^{1}$, J.C. Cruz ${ }^{1}$, T. Vilhena-Franco ${ }^{1}$, \\ W.L. Reis ${ }^{2}$, R.R. Ventura ${ }^{3}$, L.C. Reis ${ }^{4}$, L.M. Vivas ${ }^{5}$ and L.L.K. Elias ${ }^{1}$ \\ ${ }^{1}$ Departamento de Fisiologia, Faculdade de Medicina de Ribeirão Preto, Universidade de São Paulo, Ribeirão Preto, SP, Brasil \\ ${ }^{2}$ Department of Physiology, Georgia Health Sciences University, Augusta, GA, USA \\ ${ }^{3}$ Departamento de Fisiologia, Universidade Federal de Alfenas, Alfenas, MG, Brasil \\ ${ }^{4}$ Departamento de Ciências Fisiológicas, Instituto de Biologia, Universidade Federal Rural do Rio de Janeiro, Rio de Janeiro, RJ, Brasil \\ ${ }^{5}$ Instituto de Investigación Médica Mercedes y Martín Ferreyra, INIMEC-CONICET, Universidad Nacional de Córdoba \\ Córdoba, Argentina
}

\begin{abstract}
Several forebrain and brainstem neurochemical circuitries interact with peripheral neural and humoral signals to collaboratively maintain both the volume and osmolality of extracellular fluids. Although much progress has been made over the past decades in the understanding of complex mechanisms underlying neuroendocrine control of hydromineral homeostasis, several issues still remain to be clarified. The use of techniques such as molecular biology, neuronal tracing, electrophysiology, immunohistochemistry, and microinfusions has significantly improved our ability to identify neuronal phenotypes and their signals, including those related to neuron-glia interactions. Accordingly, neurons have been shown to produce and release a large number of chemical mediators (neurotransmitters, neurohormones and neuromodulators) into the interstitial space, which include not only classic neurotransmitters, such as acetylcholine, amines (noradrenaline, serotonin) and amino acids (glutamate, GABA), but also gaseous (nitric oxide, carbon monoxide and hydrogen sulfide) and lipid-derived (endocannabinoids) mediators. This efferent response, initiated within the neuronal environment, recruits several peripheral effectors, such as hormones (glucocorticoids, angiotensin II, estrogen), which in turn modulate central nervous system responsiveness to systemic challenges. Therefore, in this review, we shall evaluate in an integrated manner the physiological control of body fluid homeostasis from the molecular aspects to the systemic and integrated responses.
\end{abstract}

Key words: Hypothalamus; Gaseous neuromodulators; Neuropeptides; Endocannabinoids; Glial cells; Neurotransmitters

\section{Introduction}

In mammals, both fluid volume and osmolality levels are maintained by complex neuroendocrine, autonomic, hemodynamic, renal, and behavioral mechanisms. The harmonic operation of these systems is dependent on the presence of the following factors: 1) sensors, located at strategic positions within the central nervous system (CNS), circulatory system, liver, and kidneys, which detect changes either in the volume (mechanoreceptors: volume or pressure-receptors) or osmolality (osmo/ sodium receptors) of the extracellular fluid (ECF); 2) specific structures of the CNS responsible for the reception, analysis and integration of information and the consequent induction of appropriate responses, which are dependent on the integrity of the circuitry comprising the humoral and neural receptive areas, such as the circumventricular organs (CVOs) of the lamina terminalis [the organum vasculosum of the lamina terminalis (OVLT), the subfornical organ (SFO)], the area postrema $(\mathrm{AP})$, and the nucleus of the solitary tract (NTS), as well as areas implicated in the central integration of the visceral and somatic sensory inputs, such as the lateral parabrachial nucleus (LPBN), habenula, medial septal area (MSA), extended amygdaloid complex, paraventricular (PVN) and supraoptic nuclei (SON) of the hypothalamus, locus coeruleus (LC) and dorsal raphe nuclei (DRN), among others, and 3) the specificity of neurotransmitters, neuromodulators and neuropeptides involved in the control of sodium balance, which is 
determined by the expression of specific receptors or signaling pathways. Once activated, these systems control responses that involve the following: a) the induction of thirst, salt appetite, or both; b) changes in sympathetic activity; c) activation of local and/or systemic renin-angiotensin-aldosterone systems (RAAS); d) activation of the hypothalamic-pituitary-adrenal (HPA) axis; d) the secretion of vasopressin (AVP) and oxytocin (OT) from the neurohypophysis, and e) the production of natriuretic peptides (ANP, BNP and CNP) by the heart, endothelial cells and brain (1-3). Thus, the objective of this review is to describe the mapping and signaling of neural pathways involved in the regulation of hydromineral homeostasis, with specific emphasis on their phenotypic characterization.

\section{The anatomical basis of body fluid homeostasis: central and peripheral aspects}

Over the past decades, several studies have been conducted to identify the mechanisms and specific brain areas involved in the regulation of ECF volume and osmolality and the intake and excretion of water and electrolytes. Plasma osmolality normally ranges from 280 to $295 \mathrm{mOsm} / \mathrm{kg}$ in complex mammals and, when an increase in this parameter is detected, a number of homeostatic mechanisms, such as thirst, AVP release and renal water reabsorption, are stimulated.

Regarding these osmoregulatory mechanisms, Gilman (4) first demonstrated that cellular dehydration induced by an increase in the effective osmotic pressure or in body fluid tonicity (rather than in the absolute osmotic pressure) stimulates thirst or water intake. Ten years later, Verney (5) proposed the presence of osmoreceptors in the diencephalon, which are stimulated by the selective increase in body fluid tonicity and regulate AVP release. Since then, considerable effort has been made to precisely pinpoint their exact location. Andersson's (6) studies indicated that the rostral and medial hypothalamus (close to the midline) were the most effective sites to induce thirst after brain hypertonic sodium chloride injections. Studies of lesions in the anteroventral wall of the third ventricle (AV3V region) in goats, rats and sheep demonstrated that the ablation of this region [which is composed of several distinct brain areas, including the preoptic periventricular region, the ventral part of the median preoptic nucleus ( $\mathrm{MnPO}$ ), the anterior hypothalamic periventricular area, and the OVLT], altered water intake and AVP secretion (7-9). Evidence obtained from experiments in which sodium concentrations in the cerebrospinal fluid (CSF) were monitored during systemic infusion of hyperosmotic solutions has highlighted interest in the CVOs of the lamina terminalis as key sites for the location of osmoreceptors $(10,11)$. These results suggested that these regions would be directly influenced by
CSF tonicity (regulated by both central and peripheral administration of hyperosmotic solutions) and are consistent with the hypothesis that osmoreceptors are located in brain regions lacking the blood-brain barrier. Thus, these studies concluded that the CVOs, due to their strategic localization, would fit the requirements of being not only accessible and permeable to solutes such as glucose, sodium chloride and urea, but also capable of being influenced by ECF tonicity, thus arising as a possible anatomic locus for osmoreceptors.

In this regard, McKinley's group (12) has proposed that osmoreceptors are located specifically in the OVLT and SFO, two CVOs located at the midline of the AV3V region. Consistent with evidence provided by these studies, McKinley et al. demonstrated that lesions performed in the OVLT $(13,14)$ and SFO (15) reduced water intake and AVP release in response to systemic hypertonicity. Subsequent electrophysiological evidence showed that some cells in both CVOs altered their electrical activity in response to changes in the tonicity of their medium, being intrinsically osmosensitive because these responses were not altered by the blockade of their synaptic inputs $(16,17)$. In this context, the MnPO, which consists of the lamina terminalis, the SFO and OVLT, is also important because it receives afferent inputs from both CVOs (18), and its specific ablation disrupts osmotically induced water intake in rats (19).

Regarding the participation of the hypothalamus in these responses, one of the first hypothetical neural circuitries for the regulation of sodium balance was also established from lesion experiments. The results suggested that the hypothalamus represented the main organizer, equilibrating stimulatory and inhibitory inputs that emerged from extra-hypothalamic structures, mainly from the limbic system. According to this model, the hypothalamus is divided into two regions: the anteromedial hypothalamus $(\mathrm{AMH})$, which stimulates salt replenishment, and the anterolateral hypothalamus (ALH), which inhibits systemic sodium gain and/or prevents its loss-induced replacement. Projections from the basolateral amygdaloid complex stimulate the $\mathrm{AMH}$ and inhibit the ALH, whereas the corticomedial connections exert the opposite effects. The septal area and olfactory bulb constitute additional sources of predominantly stimulatory inputs to the inhibitory areas of both the amygdala and hypothalamus, reinforcing the inhibitory drive of salt intake pathways activated by these areas.

Therefore, the basis provided by the above mentioned pioneering studies establishing the first hypothetical neural circuitry for the regulation of fluid homeostasis has been substantially changed within the past few decades due to advances in the methodological approaches used (more restricted lesions, electrophysiological recordings, and expression of immediate-early gene products, among others). As previously mentioned, the forebrain and brainstem receptive areas as well as the 
regions involved in the integration of visceral and somatic sensory signals were also included in this circuitry. Therefore, the SFO and OVLT contain cells that are sensitive to alterations in plasma and CSF composition, such as those related to humoral signals, sodium concentrations, osmolality, and angiotensin II (ANGII) levels. Such unique features make the SFO and OVLT key brain regions to sense the status of body fluids and electrolytes. Humoral signals are then transmitted from the SFO and OVLT mainly via the MnPO to other structures in the CNS, including the hypothalamus and brainstem (3).

It has been previously demonstrated that highpressure mechanoreceptors, located not only in the systemic circulation but also in renal vessels, integrate ascending inputs that activate the brainstem and other diencephalic structures following an increase in the extracellular volume (2). Accumulating evidence indicates that the NTS is an important component of a central neural network related to the control of cardiovascular function and body fluid homeostasis, processing viscerally derived information, such as signals mediated by cardiopulmonary receptors. Noradrenergic cells of the LC are included in the pathway that modulates homeostatic responses induced by changes in the circulating volume. These connections convey information regarding the regulation of hormone secretion and the activity of the sympathetic nervous system as well as initiate behavioral adjustments with the objective of restoring body fluid homeostasis (2).

Moreover, other studies have shown that some CNS areas are also synaptically connected to the kidneys. Sly et al. (20) demonstrated that the injection of a neurotrophic virus in the kidney induces the retrograde labeling of neuronal clusters involved in homeostatic regulation. In addition to the spinal cord, several forebrain regions, including the OVLT, MnPO, SFO, the bed nucleus of the stria terminalis, anteroventral periventricular nucleus, medial and lateral preoptic areas, primary motor cortex, and the visceral area of the insular cortex, were also included in this circuitry. Moreover, renal innervation, which is considered to be entirely of sympathetic origin, participates in the homeostatic regulation of extracellular volume and osmolality by controlling three important aspects of renal function: blood flow, tubular reabsorption of electrolytes and renin secretion. In the last decade, the heart has also been shown to be an important mediator of brain-kidney integrated responses related to fluid balance. The microinjection of hypertonic saline into the AV3V region of water-loaded rats induces natriuresis and diuresis, both of which are effects attributable to the production and release of natriuretic peptides, particularly ANP, by cardiomyocytes into the systemic circulation (2). It is well known that the central ANPergic system interacts with neurohypophyseal hormones, particularly OT. This hormone, in turn, may act directly on the kidneys to promote natriuresis (an effect observed in rodents, but not in other species) as well as on cardiomyocytes to stimulate ANP production and release. Therefore, the investigations conducted thus far point to the participation of central and peripheral pathways in the control of water/ sodium retention (AVP and sympathetic nervous system) and excretion (natriuretic peptides) (2).

\section{Main neural pathways involved in the regulation of ECF volume and osmolality}

\section{The integration of forebrain sodium/osmosensitive areas and the hypothalamus via angiotensinergic, cholinergic and adrenergic pathways}

In the 1960's, Grossman (21) published the first studies that demonstrated neurotransmitter activity-controlled synaptic function in hypothalamic structures that regulate body fluid homeostasis. Moreover, these studies demonstrated that stimulation of the hypothalamus with adrenergic and cholinergic agonists induced an increase in food and water intake, respectively. Several other studies have shown that in euhydrated animals, cholinergic and angiotensinergic stimulation of the AV3V region results in a rapid increase in water intake, followed by natriuresis $(22,23)$, suggesting that cholinergic projections to the hypothalamus may represent an important pathway regulating osmotic imbalances. Subsequent studies have shown that the administration of cholinergic and alphaadrenergic agonists or antagonists into the septal area, $\mathrm{AV} 3 \mathrm{~V}$ or third ventricle modulates the natriuretic, antidiuretic and kaliuretic responses induced by carbachol. Taken together, the main findings in this area suggest that sodium/osmosensitive neurons located in the CNS modify renal sodium excretion via a stimulatory pathway involving cholinergic and alpha-adrenergic receptors and inhibit sodium excretion via a tonically active beta-adrenergicmediated pathway.

\section{The fluid balance-integrated responses of the hindbrain mediated by the serotonergic system}

Serotonin (5-HT) has been implicated in the inhibitory control of salt and water intake (24), particularly serotonergic neurons with their soma in the DRN and 5-HT terminals within the LPBN. Bilateral injections of the nonselective 5-hydroxytryptamine $\left(5-\mathrm{HT}_{1 / 2}\right)$ receptor antagonist, methysergide, into the LPBN significantly reversed the inhibitory effects of the intracerebroventricular (icv) injection of carbachol on $0.3 \mathrm{M} \mathrm{NaCl}$ intake (25). These results suggest that the LPBN integrates a satiety pathway that downregulates sodium-seeking behaviors, the mechanism of which may involve bidirectional connections with prosencephalic structures, such as the lamina terminalis, hypothalamus and brainstem.

Studies evaluating c-Fos immunoreactivity have demonstrated that the activity of serotonergic cells within 
the DRN is affected by sodium status (26). Accordingly, cFos expression was decreased in sodium-depleted animals and increased when the animals were either in balance or in the process of restoring sodium homeostasis by ingesting $0.3 \mathrm{M} \mathrm{NaCl}$ solution. $5-\mathrm{HT}$ is also released by axonal terminals and somatodendritic varicosities within the DRN, where it acts on $5-\mathrm{HT}_{1 \mathrm{~A}}$ autoreceptors to negatively modulate the serotonergic system, thereby decreasing the spontaneous firing rate of serotonergic neurons (27). These results are consistent with the concept that there is a tonic inhibition of sodium appetite exerted on the LPBN by serotonergic cells originating in the DRN. Such an inhibitory influence is likely to be reduced in a state of fluid deficiency and increased when the animals ingest water and $\mathrm{NaCl}$ to restore hydromineral balance. Such a mechanism would be important in preventing excessive $\mathrm{NaCl}$ consumption and would reduce the possibility of excess extracellular volume expansion, thus allowing a faster recovery of plasma osmolality, sodium concentration and extracellular volume during the satiety phase of sodium appetite. These changes are detected and integrated by neurons of the lamina terminalis, and then projected to the DRN via a monosynaptic connection (28).

Moreover, the serotonergic system may be subject to desensitization because an acute intra-DRN administration of 8-OH-DPAT, a 5-HT 1 A agonist, evoked an additional and significant increase in sodium intake in sodium-depleted rats, whereas repeated $8-\mathrm{OH}-\mathrm{DPAT}$ intraperitoneal or intra-DRN injections over 6 days resulted in a long-lasting reduction in cumulative sodium intake (29). According to these studies, this regulatory mechanism would be potentially associated with a reduction in $5-\mathrm{HT}_{1 \mathrm{~A}}$ receptor density or with changes in G-protein receptor coupling.

More recently, it has been demonstrated that serotonergic terminals of the LPBN originating from DRN serotonergic somas also participate in the regulation of renal and hormonal responses induced by an isotonic increase in the ECF (30). Animals with electrolytic lesions of the DRN or with serotonin depletion induced by the central administration of $p$-chlorophenylalanine display attenuated ANP release, both under resting conditions and after extracellular volume expansion (31). Taken together, these behavioral and neuroendocrine observations suggest that the serotonergic system acts via differential projecting pathways and receptor mechanisms to induce sodium satiety, which may include 1) a barosensitive mechanism; 2) a reduced aversion to salt during hyponatremia, as deduced from evidence suggesting the presence of specific sodium channels in the SFO, and 3) elevated plasma ANGII concentrations, which may sensitize the CVOs $(1,3,32)$.

\section{The midbrain and hindbrain GABAergic systems}

GABA, the major inhibitory neurotransmitter in the mammalian CNS, mediates the inhibitory actions of interneurons in the brain, as well as the presynaptic inhibition in the spinal cord. The main ionotropic subtype of GABA receptors in the brain is $\mathrm{GABA}_{A}$, which consists of a ligand-operated chloride ion channel. This channel is opened in response to GABA release by presynaptic neurons, mediating phasic inhibition, or following the activation of extrasynaptic receptors, mediating tonic inhibition of the postsynaptic cell (33).

It has been shown that inhibitory mechanisms in the LPBN and central GABAergic mechanisms are involved in the regulation of behavioral responses particularly related to water and sodium intake. The activation of $G_{A B A}$ receptors by muscimol (a $\mathrm{GABA}_{\mathrm{A}}$ agonist) injected into the LPBN induces the ingestion of water and $0.3 \mathrm{M} \mathrm{NaCl}$ solution in both euhydrated and hyperosmotic celldehydrated rats, with the effect on sodium intake being reversed by the administration of bicuculline, a $G_{A B A}$ antagonist (34).

Furthermore, GABA plays a major role in the control of neurohypophyseal output because both GABA and muscimol decrease hypertonicity-induced AVP release (35). GABA also reduces the firing rate of AVP-secreting neurons when applied microionophoretically into the SON of rats (36). Thus, many questions remain unanswered regarding how an increase in volume affects neurohypophyseal hormone secretion and which neural pathways are responsible for conveying the signal from cardiac and arterial baroreceptors to the hypothalamus. GABAergic nerve terminals have been observed in neurosecretory nerve endings in the neurohypophysis (37), suggesting an important role for the inhibitory neurotransmitter GABA in regulating the activity of OT and AVP neurons. It is known that extracellular volume expansion (EVE) activates CNS regions associated with the inhibition of AVP release and sympathetic nerve activity and stimulates OT secretion (2). Consistent with these findings, it has been proposed that the perinuclear zone of the SON, a GABAergic region surrounding this hypothalamic nucleus, constitutes the major source of inhibition in AVP neurons following an acute increase in blood pressure, thus decreasing the activity of AVP-secreting cells of the SON in response to stretching of the caval-atrial junction (38).

Other sources of GABAergic inputs to the hypothalamic neurohypophyseal system have also been described, including the amygdaloid complex. The central amygdaloid nucleus (CeA) and the bed nucleus of the stria terminalis (BNST), which is considered to be a component of the "extended amygdala", establish important connections with the hypothalamus and other brain areas controlling visceral and sensory information. Furthermore, increases in urine output, renal sodium excretion, and plasma OT and ANP concentrations induced by isotonic and hypertonic EVE are attenuated in rats pretreated with muscimol into the CeA. Bicuculline, an antagonist of $\mathrm{GABA}_{\mathrm{A}}$ receptors, injected into the $\mathrm{CeA}$, 
also reduced the effects of hypertonic EVE on urinary volume and ANP secretion (39). Thus, taken together, these data suggest that the CeA GABAergic mechanisms are involved in the control of ANP and OT secretion, as well as in the control of sodium and water excretion in response to isotonic and hypertonic increases in the ECF.

Furthermore, icv injection of GABA agonists decreases arterial blood pressure, heart rate and sympathetic nerve activity, while icv injections of GABA antagonists such as bicuculline markedly increase arterial blood pressure and heart rate due to an increase in sympathetic nerve activity (40). Accumulating evidence supports a blunted GABAergic inhibition within the PVN as a critical mechanism contributing to sympathoexcitation in cardiovascular-related disorders, including hypertension (41). Inhibition of neuronal excitability in hypothalamic oxytocinergic and vasopressinergic neurons by nitric oxide (NO) involves pre- and postsynaptic potentiation of GABAergic synaptic activity in the SON (42). Numerous studies have shown that the neuronal activity in the PVN, as well as the sympathetic outflow from the PVN, is basally restrained by a GABAergic inhibitory tone (43). Taken together, these results suggest that GABA activity or its interaction with other neuromodulators is dependent on a particular physiological condition and may result in either a positive or negative feedback compensatory mechanism to coordinate complex neuroendocrine, behavioral and autonomic output patterns.

\section{The local modulation of synaptic function}

The understanding of the complex central circuitries involved in the control of hydromineral balance was dramatically increased after the discovery that the CNS produces several peptides/hormones that were primarily identified in the periphery. Accordingly, neurons not only express several receptor systems but also synthesize hormones that are released into the interstitial space where they modulate synaptic function in an autocrine or paracrine manner. This mechanism is supported by evidence of the local expression of specific enzymatic pathways and precursors. In the following sections, this review will address the contribution of local modulators of synaptic function to the control of water and sodium balance, as well as introduce glial cells and gliotransmitters as active elements within this context.

Neuropeptides. ANGII, when injected into specific areas of the CNS, exhibits dipsogenic and natriorexigenic effects. It has been well demonstrated that the AV3V region robustly expresses not only type 1 (AT1) ANGII receptors, which are responsible for most of the ANGIImediated behavioral effects, but also angiotensinergic terminals. This peptide is produced in the CNS independently of the circulating RAAS, which raises the possibility that endogenous brain-derived ANGII may be involved in the physiological regulation of water and salt intake; although there have been no conclusive demonstrations. Conversely, the widespread distribution of ANGII receptors throughout the CNS suggests that this locally produced peptide may have a more complex function not related to the control of water and salt appetite $(1,3)$. The presence of ANP-secreting neurons and ANP receptors in CNS structures related to the homeostatic control of hydromineral homeostasis and cardiovascular function has also been demonstrated (44). It has been reported that ANP or CNP injected into the AV3V region decreases plasma ANP concentration in volumeexpanded rats, with no change in mean arterial pressure or heart rate (45). Thus, local increases in ANP within the CNS elicited by EVE might constitute a feedback loop that inhibits ANP neuronal activity (2).

Autocrine mechanisms have also been shown to regulate the activity of the magnocellular neurosecretory system. Consequently, OT is released from somatodendrites of SON magnocellular neurons and acts on presynaptic autoreceptors to facilitate the release of endocannabinoids (ECBs) and GABA, which in turn inhibit neuronal activity (46). Furthermore, the hypoosmolalityinduced somatodendritic release of AVP by SON neurons and the local activation of type 2 AVP (V2) receptors have been recently implicated in the mediation of cell volume regulation in vitro (47).

Gaseous neuromodulators. Soluble gases have a short half-life, which limits their actions on neighboring cells that express or are in close proximity to the enzymatic machinery responsible for their synthesis. Once produced, these highly membrane-permeable molecules are rapidly converted into ineffective products by specific chemical reactions. The neuronal isoform of NO synthase enzyme (nNOS), which is responsible for the production of $\mathrm{NO}$ within the CNS, is expressed in several cell clusters, including the lamina terminalis, magnocellular neurosecretory cells of the SON and PVN nuclei and the posterior pituitary (48). Furthermore, NO modulates the activity of the magnocellular neurosecretory system. Rats that are centrally or peripherally injected with L-NAME, a non-selective NOS inhibitor, show increased plasma AVP and OT concentrations (49). Interestingly, upregulation of NOS mRNA and NOS activity is induced in rats subjected to chronic salt loading (50), suggesting that mechanisms responsible for the prevention of hormonal depletion may be activated after prolonged exposure to these life-limiting conditions.

Several studies using extracellular and intracellular recordings have demonstrated that the NO precursor Larginine decreases, whereas L-NAME increases, the firing frequency of magnocellular neurosecretory cells, suggesting a tonic inhibitory effect on neuronal activity (51). Accordingly, NO increases the amplitude and frequency of GABA miniature inhibitory postsynaptic currents in SON magnocellular neurons, suggesting that NO inhibition of SON neuronal excitability involves 
pre- and postsynaptic potentiation of GABAergic synaptic activity (42).

Peripherally, the secretion of ANP by the heart is also modulated by local NO production. The main findings reported so far have supported an inhibitory role for NO in ANP release, a mechanism that seems to be disrupted and to contribute to the development of heart failure (52). In the kidneys, NOS is present in vascular and tubular elements, and sustained changes in NO synthesis have been implicated in some pathological conditions, such as glomerulonephritis and chronic kidney disease (53).

Carbon monoxide (CO) is another gaseous modulator with potential effects on body fluid homeostasis. It is one of the most toxic molecules in nature, and its main biological effect consists of activating cyclic guanosine monophosphate production, with reported effects on synaptic plasticity and neuromodulation (54). The discovery of high levels of heme oxygenase $(\mathrm{HO})$ in the brain, however, strongly suggested a role for $\mathrm{CO}$ mediated signaling in central physiological functions. In the hypothalamus, a recent study showed that immunoreactivity for type $1 \mathrm{HO}(\mathrm{HO}-1)$ in both vasopressinergic and oxytocinergic neurons is increased by water deprivation (55). Accordingly, inhibition of $\mathrm{HO}$ was shown to decrease the hyperosmolality-induced release of OT and ANP by the medial basal hypothalamus in vitro (56). Similarly, inhibition of this enzyme also induced a strong hyperpolarization and a decrease in the firing rate of magnocellular neurosecretory cells in water-deprived animals (55). In addition to acting individually, CO has also been shown to interact with the nitrergic system to control hydromineral homeostasis. Moreover, use of an $\mathrm{HO}$ inhibitor increases the production of L-citrulline, a coproduct of NO synthesis, by the medial basal hypothalamus in vitro in response to hyperosmolality (56). Thus, CO appears to play a predominantly facilitatory role in AVP and OT release, although most studies suggest that NO has an opposite effect, inhibiting AVP and OT release. On the basis of the evidence that the final products of both NOergic and COergic systems can reciprocally affect enzymatic activity, it can be suggested that these two systems integrate to form a feedback loop implicated in the local control of hypothalamic neurosecretory system output.

The last gaseous neuromodulator to be described was $\mathrm{H}_{2} \mathrm{~S}$, which is endogenously produced in relatively large amounts by the CNS. The enzymes cystathionine- $\beta$ synthase and cystathionine- $\gamma$-lyase are responsible for $\mathrm{H}_{2} \mathrm{~S}$ synthesis from L-cysteine. Both the expression and activity of these isoforms are known to be modulated by other signaling molecules such as $\mathrm{Ca}^{2+}$, as well by circulating hormones such as insulin, glucagon and glucocorticoids (57). Although experimental evidence linking $\mathrm{H}_{2} \mathrm{~S}$ to hydromineral homeostatic control has not yet been reported, a potential role for this nonconventional neurotransmitter in the regulation of neuroendocrine output could be speculated because $\mathrm{H}_{2} \mathrm{~S}$ has been shown to interact with $\mathrm{NO}$ and $\mathrm{CO}(57)$.

Endocannabinoids. In the past few years, endogenously produced cannabinoids have emerged as important signaling molecules mediating several physiological processes, particularly the control of energy homeostasis. The main ECBs that have been characterized thus far include anandamide and 2-arachidonoylglycerol, which are produced on demand by membrane phospholipids. A receptor for cannabis-like substances (CB1R) was first identified in 1988. The type 2 cannabinoid receptor (CB2R) was characterized a few years later and exhibits a very distinct expression profile. The physiological actions of the ECB system are produced mostly by the interaction of endogenous ligands with CB1R and/or CB2R. However, the involvement of orphan receptors, such as GPR55, in the mediation of some responses still remains to be examined.

CB1R is a G-protein coupled receptor (GPCR) whose activation affects neurotransmission and signal transduction. Most of its previously described effects are related to 1 ) inhibition of the adenylyl cyclase-mediated pathway, 2) activation of potassium channels, and 3) inhibition of voltage-gated calcium channels (58). Over the past few years, the identification of potential antagonists for the ECB system has been of great interest since CB1R was identified as an important target in the control of metabolic diseases, particularly those related to increased abdominal adiposity. However, the assumption that GPCRs, including CB1R, can assume multiple conformations and affinity states has dramatically increased the possibilities by which potential drugs can affect CB1R-mediated signaling. Thus, several studies have suggested that the ECB system may be active under basal conditions. The constant production and release of ECBs, the presence of constitutively activated $\mathrm{CB} 1 \mathrm{R}$, and the presence or absence of relatively low concentrations of the endogenous ligands would contribute to this basal activity (59).

An emerging role for ECBs consists of their mediation of glucocorticoid-related effects, particularly in the CNS. Activation of the HPA axis, with the consequent release of glucocorticoids, is known to exert an inhibitory effect on the activation of most neuroendocrine functions, including the synthesis/secretion of neurohypophyseal peptides, particularly OT (60). Nongenomic signaling pathways activated by the binding of glucocorticoids to conserved cell membrane sites have been characterized, and this mechanism has been implicated in the rapid changes observed in neurotransmission following an increase in circulating glucocorticoid levels.

The local production and release of ECBs within the PVN and SON in the presence of glucocorticoids results in decreased glutamatergic and increased GABAergic inputs to both parvocellular and magnocellular neurons in vitro (61). In addition to directly stimulating GABA neurotransmission, glucocorticoids also stimulate NO 
release, which potentiates the negative drive to these cell groups. CB1R is the main isoform involved in these responses because rimonabant, a CB1R antagonist, potentiates the secretion of AVP and OT induced by a hypertonic EVE, as well as reverses the inhibitory effects of dexamethasone on hormonal release under the same experimental conditions (62). Thus, together with gaseous neuromodulators, locally produced ECB are important signaling molecules that control hypothalamic function in situ.

Glial cells and gliotransmitters. It has been proposed that the SON exhibits an intrinsic osmosensitivity, which is provided by changes in mechanosensitive cationic channels. These channels depolarize and hyperpolarize magnocellular neurons that are exposed to hyperosmotic or hypoosmotic conditions (63). Recent studies have identified an additional osmoregulatory mechanism within the SON involving astroglia, through which astrocytes may regulate magnocellular excitability via gliotransmitter release and neurotransmitter clearance and act as osmosensory elements $(31,64-67)$. Several studies have demonstrated that taurine, an organic osmolyte, is present in glial processes surrounding magnocellular neurons. Moreover, several reports have also shown that certain stimuli, such as hypotonicity, can induce an increase in taurine release by astrocytes. This effect, in turn, was shown to inhibit $\mathrm{Ca}^{2+}$ influx and contribute to a decrease in AVP and OT release (64). Furthermore, recent studies have demonstrated that astrocytes of the SON are activated in response to acute exposure to hypertonic saline, promoting glutamate release through connexin-43 functional hemi-channels, which are the predominant gap junction proteins expressed in astrocytes, and subsequently activating magnocellular neurons $(65,67)$. Furthermore, several studies have reported that dehydration decreases the expression of type 1 glutamate transporters in SON astrocytes (68), suggesting a diminished astrocytic glutamate uptake with a consequent increase in glutamate availability in the extracellular space, which may result in the tonic activation of presynaptic glutamate receptors on neuronal terminals. Because dehydration induces morphological changes within the hypothalamus, this process may result in the reduction of astrocyte-mediated exposure of magnocellular neurons (69). Previous studies conducted by Boudaba et al. (68) argue that this dehydration-induced increase in extracellular glutamate accumulation could also be modulated by the retraction of astroglial processes around SON neurons, resulting in direct and extensive juxtapositions of magnocellular neuronal surfaces.

Moreover, several studies have hypothesized that $\mathrm{Na}_{\mathrm{x}}$, an atypical sodium channel, may constitute an important sodium sensor within the CNS $(32,70,71)$. Watanabe et al. (70) demonstrated that salt-aversive behaviors induced by dehydration were abolished in $\mathrm{Na}_{x}^{-/-}$ transgenic mice. Moreover, infusion of either mannitol or isotonic saline into the cerebral ventricle induced similar behavioral effects on water and sodium intake in $\mathrm{Na}_{\mathrm{x}}{ }^{+/+}$ and $\mathrm{Na}_{x}{ }^{-1-}$ mice, suggesting that $\mathrm{Na}_{x}$ is particularly sensitive to extracellular sodium concentrations. Furthermore, these studies also showed that the $\mathrm{Na}_{x}$ channel is expressed in the perineuronal processes of astrocytes and ependymal cells in sensory CVOs. The mechanism by which astrocytes are activated by increased ECF sodium concentrations was recently demonstrated by Shimizu and his group (71), who revealed a dehydration-induced opening of $\mathrm{Na}_{\mathrm{x}}$ channels, which resulted in 1) increased intracellular sodium concentrations, 2) increased $\mathrm{Na}^{+} / \mathrm{K}^{+}$-ATPase activity and ATP expenditure, and 3) stimulation of anaerobic glucose metabolism and lactate release by astrocytes, supplying SON neurons with this energy source. Furthermore, the same group has demonstrated that the dehydration-induced lactate release by astrocytes activates SFO GABAergic neurons via the production of ATP, with this mechanism presumably being implicated in the control of salt intake.

Recent functional experiments performed by our group have also demonstrated that the central administration of ANGIl stimulates AVP and OT release (49). It has been previously reported that both AT1 and AT2 are widely distributed in the hypothalamus, as they are expressed in both neuronal cell types and in astrocytes (72). This study also demonstrated that AT2 receptors are expressed in astrocytes juxtaposed to magnocellular neurons in the PVN. Although these studies have suggested that centrally produced ANGII activity on hypothalamic astrocytes may play an important role in hydromineral balance, the mechanisms underlying these effects still remain unclear.

\section{Peripheral hormones alter the responsiveness of the CNS to body fluid disturbances: focus on estrogen actions}

The first studies that proposed that gonadal hormones are involved in the control of hydromineral balance stemmed from data showing that water intake and sodium appetite varied along the estrous cycle. Accordingly, studies by Antunes-Rodrigues et al. (73) reported a spontaneous decrease in sodium intake during estrus, which is the stage when the female reproductive cycle undergoes high plasma estrogen concentrations, and an increase in sodium intake during diestrus, when low plasma levels of estrogen are detected. Treatment with estradiol attenuates water intake in OVX animals receiving hyperoncotic colloid (74) or isoproterenol and hypertonic saline (75), or animals subjected to water deprivation (76). Other groups have reported that estrogen therapy decreases not only water intake but also abolishes salt 
ingestion induced by central ANGII administration in OVX rats (77).

More recently, the participation of the RAAS in the mediation of the behavioral responses induced by estradiol has been demonstrated. Estradiol was shown to inhibit ANGII AT1 receptors and angiotensin-converting enzyme (ACE) expression in the SFO (78). Furthermore, it was also demonstrated by our group that the central AT1 blockade in OVX rats inhibits 1) the dipsogenic response induced by water deprivation, osmotic stimulation, chronic sodium depletion and furosemide + captopril (furo + cap) experimental models and 2) the natriorexigenic response induced by sodium depletion $(77,79)$. These studies also demonstrated that estrogen significantly attenuates the losartan inhibition of thirst and sodium appetite driven by sodium depletion and furo + cap experimental models. Consistent with recent evidence from the literature suggesting a potentially protective effect of estradiol on the cardiovascular system, these observations further support a role for this hormone in the behavioral control of water and salt intake because of the clearly demonstrated influence of estrogenic status on the AT1-mediated behavioral responses.

In addition to the modulation of several mechanisms involved in the induction of drinking behavior, estrogen also modulates inhibitory serotonergic mechanisms controlling salt intake. Several studies have demonstrated that estrogen modulates the basal firing rate of serotonergic neurons (80) and enhances serotonin transporter mRNA expression in the DRN of OVX animals (81). Furthermore, when estrogen is chronically administered to OVX rats, an increase in the mRNA expression for tryptophan hydroxylase-2 is observed in the DRN (82). More recently, it was also demonstrated that the estrogen-dependent inhibition of sodium appetite in both normal cycling rats and OVX animals with estradiol replacement may involve an interaction between excitatory neurons of the OVLT and the inhibitory serotonergic drive of the DRN, which are key pathways that underlie the responses to hyponatremia and hypovolemia (83).

Further effects of estrogens on fluid balance involve the control of neurohypophyseal hormones and ANP. Changes in plasma AVP during the reproductive cycle have been previously reported (84). Furthermore, several studies have proposed a stimulatory role for estradiol on OT and ANP secretion $(85,86)$. More recently, the contribution of estradiol to AVP, OT and ANP secretion as well as to vasopressinergic and oxytocinergic neuronal activation has been demonstrated $(87,88)$. Estradiol has been shown to positively modulate AVP and OT secretion in response to osmotic stimulation induced by hypertonic extracellular volume expansion (87) and hypovolemic shock (88), without altering ANP secretion. These same studies also showed that the activity of both oxytocinergic and vasopressinergic neurons in the SON and PVN may be modulated by estradiol.

Taken together, these findings are strongly supported by the evidence that estradiol receptor beta $(E R-\beta)$ is expressed in the SON and PVN, where it co-localizes with AVP and OT (89), and the ER alpha (ER- $\alpha)$ is expressed in afferent osmosensitive neurons located in the SFO and OVLT that project to the SON and PVN (90). Thus, modulation exerted by estradiol on vasopressinergic and oxytocinergic activity could be produced by a direct effect because PVN and SON neurons express ER- $\beta$, and/or by an indirect mechanism of action presumably arising from the lamina terminalis. In this context, the hyperosmolalityinduced activation of first-order ER- $\alpha$-expressing neurons of the CVOs could produce a subsequent activation of vasopressinergic and oxytocinergic neurons in the SON and PVN. This latter hypothesis is supported by a recent study showing that AVP immunoreactivity in the SON and $\mathrm{PVN}$ is significantly elevated in response to treatment with an ER- $\alpha$ agonist, but not with an ER- $\beta$ agonist, suggesting that estradiol may regulate vasopressinergic neuronal activity by acting on afferent neurons expressing ER- $\alpha$. Furthermore, the hypovolemia-induced activation of central areas controlling plasma volume and arterial pressure, such as the SFO, may also be modulated by estradiol.

Taken together, these data indicate that estradiol participates in the control of fluid balance by the following mechanisms: 1) increasing the responsiveness of vasopressinergic and oxytocinergic neurons in the PVN and SON as well as the secretion of the AVP and OT neuropeptides; 2) increasing the release of natriuretic factors (ANP and OT) in response to osmotic stimulation or other systemic challenges, 3) decreasing brain ANGII responsiveness via the modulation of AT1-mediated signaling, and 4) increasing the DRN serotonergic activity, which consequently inhibits salt appetite.

\section{Conclusions and Perspectives}

The identification and the characterization of the phenotypical pathways activated in the CNS in response to peripherally produced signals have significantly improved our understanding of the redundant mechanisms controlling body fluid homeostasis. These pathways are mediated not only by classic neurotransmitters such as acetylcholine, amines (noradrenaline, serotonin) and amino acids (glutamate, GABA), but also by gaseous (NO, $\mathrm{CO}$ and $\mathrm{H}_{2} \mathrm{~S}$ ) and lipid-derived (ECBs) mediators. The efferent responses initiated within the neuronal environment and modulated by glial cells recruit several peripheral effectors (such as glucocorticoids, ANGII, estrogen), which in turn modulate central responsiveness to systemic challenges. Since most of these hormones produce a broad range of effects in the CNS, the future directions in this field are now being aimed at 1) the identification of specific signaling pathways that may characterize site-specific actions, as well as 2) the in situ 
manipulation of these cascades in different brain areas. This latter objective may be accomplished, among other methodological strategies, by the use of local modulators of gene activity, such as viral vectors, which induce in vivo gene transfer, down- or upregulating endogenous gene function. Furthermore, the evidence that these brain networks can be affected by plasticity has also focused attention on the importance of fetal programming and

\section{References}

1. Fitzsimons JT. Angiotensin, thirst, and sodium appetite. Physiol Rev 1998; 78: 583-686.

2. Antunes-Rodrigues $\mathrm{J}$, de Castro $\mathrm{M}$, Elias LL, Valenca MM, McCann SM. Neuroendocrine control of body fluid metabolism. Physiol Rev 2004; 84: 169-208, doi: 10.1152/physrev. 00017.2003

3. McKinley MJ, Johnson AK. The physiological regulation of thirst and fluid intake. News Physiol Sci 2004; 19: 1-6.

4. Gilman A. The relation between blood osmotic pressure, fluid distribution and voluntary water intake. Am J Physiol 1937; 120: 323-328.

5. Verney EB. The antidiuretic hormone and the factors which determine its release. Proc R Soc Lond B Biol Sci 1947; 135: 25-106, doi: 10.1098/rspb.1947.0037.

6. Andersson B. The effect of injections of hypertonic $\mathrm{NaCl}-$ solutions into different parts of the hypothalamus of goats. Acta Physiol Scand 1953; 28: 188-201, doi: 10.1111/j.1748-1716. 1953.tb00969.x.

7. Andersson B, Leksell LG, Lishajko F. Perturbations in fluid balance induced by medially placed forebrain lesions. Brain Res 1975; 99: 261-275, doi: 10.1016/0006-8993(75)90028-1.

8. Buggy J, Jonhson AK. Preoptic-hypothalamic periventricular lesions: thirst deficits and hypernatremia. Am J Physiol 1977; 233: R44-R52.

9. McKinley MJ, Denton DA, Leventer M. Adipsia in sheep caused cerebral lesions. In: de Caro G, Massi M, Epstein AN (Editors), The physiology of thirst and sodium appetite. New York: Plenum Press; 1986. p 321-326.

10. McKinley MJ, Denton DA, Weisinger RS. Sensors for antidiuresis and thirst - osmoreceptors or CSF sodium detectors? Brain Res 1978; 141: 89-103, doi: 10.1016/0006-8993(78)90619-4.

11. Thrasher TN, Jones RG, Keil LC, Brown CJ, Ramsay DJ. Drinking and vasopressin release during ventricular infusions of hypertonic solutions. Am J Physiol 1980; 238: R340-R345.

12. McKinley MJ, Denton DA, Leksell LG, Mouw DR, Scoggins BA, Smith $\mathrm{MH}$, et al. Osmoregulatory thirst in sheep is disrupted by ablation of the anterior wall of the optic recess. Brain Res 1982; 236: 210-215, doi: 10.1016/0006-8993(82)90048-8.

13. Thrasher TN, Keil LC, Ramsay DJ. Lesions of the organum vasculosum of the lamina terminalis (OVLT) attenuate osmotically-induced drinking and vasopressin secretion in the dog. Endocrinology 1982; 110: 1837-1839, doi: 10.1210/ endo-110-5-1837.

14. Sayer RJ, Hubbard JI, Sirett NE. Rat organum vasculosum laminae terminalis in vitro: responses to transmitters. $A m \mathrm{~J}$ Physiol 1984; 247: R374-R379.

15. Mangiapane ML, Thrasher TN, Keil LC, Simpson JB, Ganong WF. Role for the subfornical organ in vasopressin aging-related changes for the integrity of homeostatic responses regarding hydromineral balance.

\section{Acknowledgments}

Research supported by FAPESP (\#2008/50611-8), CNPq (\#301827/2011/7), CONICET, ANPCyT, and SECYT.

release. Brain Res Bull 1984; 13: 43-47, doi: 10.1016/03619230(84)90006-6.

16. Vivas L, Chiaraviglio E, Carrer HF. Rat organum vasculosum laminae terminalis in vitro: responses to changes in sodium concentration. Brain Res 1990; 519: 294-300, doi: 10.1016/0006-8993(90)90091-O.

17. Sibbald JR, Hubbard JI, Sirett NE. Responses from osmosensitive neurons of the rat subfornical organ in vitro. Brain Res 1988; 461: 205-214, doi: 10.1016/0006-8993(88)90251-X.

18. Miselis RR. The efferent projections of the subfornical organ of the rat: a circumventricular organ within a neural network subserving water balance. Brain Res 1981; 230: 1-23, doi: 10.1016/0006-8993(81)90388-7.

19. Mangiapane ML, Thrasher TN, Keil LC, Simpson JB, Ganong WF. Deficits in drinking and vasopressin secretion after lesions of the nucleus medianus. Neuroendocrinology 1983; 37: 73-77, doi: 10.1159/000123518.

20. Sly JD, Colvill L, McKinley JM, Oldfield JB. Identification of neural projections from the forebrain to the kidney, using the virus pseudorabies. J Auton Nerv Syst 1999; 77: 73-82, doi: 10.1016/S0165-1838(99)00031-4.

21. Grossman SP. Eating or drinking elicited by direct adrenergic or cholinergic stimulation of hypothalamus. Science 1960; 132: 301-302, doi: 10.1126/science.132.3422.301.

22. Antunes-Rodrigues J, McCann SM. Water, sodium chloride, and food intake induced by injections of cholinergic and adrenergic drugs into the third ventricle of the rat brain. Proc Soc Exp Biol Med 1970; 133: 1464-1470.

23. Routtenberg A, Simpson JB. Carbachol-induced drinking at ventricular and subfornical organ sites of application. Life Sci 1971; 10: 481-490, doi: 10.1016/0024-3205(71)90210-4

24. Reis LC. Role of the serotoninergic system in the sodium appetite control. An Acad Bras Cienc 2007; 79: 261-283, doi: 10.1590/S0001-37652007000200009.

25. Menani JV, Barbosa SP, De Luca LA Jr, De Gobbi JI, Johnson AK. Serotonergic mechanisms of the lateral parabrachial nucleus and cholinergic-induced sodium appetite. Am J Physiol Regul Integr Comp Physiol 2002; 282: R837-R841.

26. Franchini LF, Johnson AK, de Olmos J, Vivas L. Sodium appetite and Fos activation in serotonergic neurons. $A m \mathrm{~J}$ Physiol Regul Integr Comp Physiol 2002; 282: R235-R243.

27. Blier $P$, Pineyro G, el Mansari M, Bergeron R, de Montigny C. Role of somatodendritic 5-HT autoreceptors in modulating 5-HT neurotransmission. Ann N Y Acad Sci 1998; 861: 204-216, doi: 10.1111/j.1749-6632.1998.tb10192.x.

28. Badaue-Passos D Jr, Godino A, Johnson AK, Vivas L, Antunes-Rodrigues $\mathrm{J}$. Dorsal raphe nuclei integrate allostatic information evoked by depletion-induced sodium 
ingestion. Exp Neurol 2007; 206: 86-94, doi: 10.1016/ j.expneurol.2007.04.008.

29. Fonseca FV, Mecawi AS, Araujo IG, Almeida-Pereira G, Magalhaes-Nunes AP, Badaue-Passos D Jr, et al. Role of the $5-\mathrm{HT}(1 \mathrm{~A})$ somatodendritic autoreceptor in the dorsal raphe nucleus on salt satiety signaling in rats. Exp Neurol 2009; 217: 353-360, doi: 10.1016/j.expneurol.2009.03.015.

30. Margatho LO, Giusti-Paiva A, Menani JV, Elias LL, Vivas LM, Antunes-Rodrigues J. Serotonergic mechanisms of the lateral parabrachial nucleus in renal and hormonal responses to isotonic blood volume expansion. $A m \mathrm{~J}$ Physiol Regul Integr Comp Physiol 2007; 292: R1190R1197, doi: 10.1152/ajpregu.00351.2006.

31. Reis LC, Ramalho MJ, Favaretto AL, Gutkowska J, McCann SM, Antunes-Rodrigues J. Participation of the ascending serotonergic system in the stimulation of atrial natriuretic peptide release. Proc Natl Acad Sci U S A 1994; 91: 1202212026, doi: 10.1073/pnas.91.25.12022.

32. Noda M. Hydromineral neuroendocrinology: mechanism of sensing sodium levels in the mammalian brain. Exp Physiol 2007; 92: 513-522, doi: 10.1113/expphysiol.2006.035659.

33. Farrant $M$, Nusser $Z$. Variations on an inhibitory theme: phasic and tonic activation of $\operatorname{GABA}(\mathrm{A})$ receptors. Nat Rev Neurosci 2005; 6: 215-229, doi: 10.1038/nrn1625.

34. Kimura EH, Oliveira LB, Colombari DS, De Luca LA Jr, Menani JV, Callera JC. Sodium intake by hyperosmotic rats treated with a $\operatorname{GABA}(\mathrm{A})$ receptor agonist into the lateral parabrachial nucleus. Brain Res 2008; 1190: 86-93, doi: 10.1016/j.brainres.2007.11.004.

35. Iovino M, de Caro G, Massi M, Steardo L, Poenaru S. Muscimol inhibits ADH release induced by hypertonic sodium chloride in rats. Pharmacol Biochem Behav 1983; 19: 335-338, doi: 10.1016/0091-3057(83)90061-8.

36. Arnauld E, Cirino M, Layton BS, Renaud LP. Contrasting actions of amino acids, acetylcholine, noradrenaline and leucine enkephalin on the excitability of supraoptic vasopressin-secreting neurons. A microiontophoretic study in the rat. Neuroendocrinology 1983; 36: 187-196, doi: 10.1159/ 000123455

37. Oertel WH, Mugnaini E, Tappaz ML, Weise VK, Dahl AL, Schmechel DE, et al. Central GABAergic innervation of neurointermediate pituitary lobe: biochemical and immunocytochemical study in the rat. Proc Natl Acad Sci U S A 1982; 79: 675-679, doi: 10.1073/pnas.79.2.675.

38. Grindstaff RR, Grindstaff RJ, Cunningham JT. Effects of right atrial distension on the activity of magnocellular neurons in the supraoptic nucleus. Am J Physiol Regul Integr Comp Physiol 2000; 278: R1605-R1615.

39. Margatho LO, Elias LL, Antunes-Rodrigues J. GABA in the central amygdaloid nucleus modulates the electrolyte excretion and hormonal responses to blood volume expansion in rats. Braz J Med Biol Res 2009; 42: 114-121, doi: 10.1590/S0100-879X2009000100016.

40. Sun AY, Li DX. Cardiovascular responses to intracerebroventricular injection of GABA in renovascular hypertensive rats. Zhongguo Yao Li Xue Bao 1994; 15: 136-138.

41. Li DP, Pan HL. Plasticity of GABAergic control of hypothalamic presympathetic neurons in hypertension. $\mathrm{Am}$ J Physiol Heart Circ Physiol 2006; 290: H1110-H1119, doi: 10.1152/ajpheart.00788.2005.

42. Stern JE, Ludwig M. NO inhibits supraoptic oxytocin and vasopressin neurons via activation of GABAergic synaptic inputs. Am J Physiol Regul Integr Comp Physiol 2001; 280: R1815-R1822.

43. Yang $\mathrm{Z}$, Coote $\mathrm{JH}$. Role of GABA and $\mathrm{NO}$ in the paraventricular nucleus-mediated reflex inhibition of renal sympathetic nerve activity following stimulation of right atrial receptors in the rat. Exp Physiol 2003; 88: 335-342, doi: 10.1113/eph8802561.

44. Quirion R, Dalpe M, DeLean A. Characterization, distribution and plasticity of atrial natriuretic factor binding sites in brain. Can J Physiol Pharmacol 1988; 66: 280-287, doi: 10.1139/y88-048.

45. Puyo AM, Vatta MS, Donoso AS, Bianciotti LG, Fernandez $\mathrm{BE}$. Central natriuretic peptides regulation of peripheral atrial natriuretic factor release. Regul Pept 2000; 90: 93-99, doi: 10.1016/S0167-0115(00)00115-4.

46. Oliet SH, Baimoukhametova DV, Piet R, Bains JS. Retrograde regulation of GABA transmission by the tonic release of oxytocin and endocannabinoids governs postsynaptic firing. J Neurosci 2007; 27: 1325-1333, doi: 10.1523/JNEUROSCI.2676-06.2007.

47. Sato K, Numata T, Saito T, Ueta Y, Okada Y. V(2) receptormediated autocrine role of somatodendritic release of AVP in rat vasopressin neurons under hypo-osmotic conditions. Sci Signal 2011; 4: ra5, doi: 10.1126/scisignal.2001279.

48. Bredt DS, Glatt CE, Hwang PM, Fotuhi M, Dawson TM, Snyder $\mathrm{SH}$. Nitric oxide synthase protein and mRNA are discretely localized in neuronal populations of the mammalian CNS together with NADPH diaphorase. Neuron 1991; 7: 615-624, doi: 10.1016/0896-6273(91)90374-9.

49. Reis WL, Giusti-Paiva A, Ventura RR, Margatho LO, Gomes DA, Elias LL, et al. Central nitric oxide blocks vasopressin, oxytocin and atrial natriuretic peptide release and antidiuretic and natriuretic responses induced by central angiotensin II in conscious rats. Exp Physiol 2007; 92: 903-911, doi: 10.1113/expphysiol.2007.037911.

50. Kadowaki K, Kishimoto J, Leng G, Emson PC. Upregulation of nitric oxide synthase (NOS) gene expression together with NOS activity in the rat hypothalamo-hypophysial system after chronic salt loading: evidence of a neuromodulatory role of nitric oxide in arginine vasopressin and oxytocin secretion. Endocrinology 1994; 134: 10111017, doi: 10.1210/en.134.3.1011.

51. Ventura RR, Aguiar JF, Antunes-Rodrigues J, Varanda WA Nitric oxide modulates the firing rate of the rat supraoptic magnocellular neurons. Neuroscience 2008; 155: 359-365, doi: 10.1016/j.neuroscience.2008.06.005.

52. Dietz JR. Mechanisms of atrial natriuretic peptide secretion from the atrium. Cardiovasc Res 2005; 68: 8-17, doi: 10.1016/j.cardiores.2005.06.008.

53. Baylis $\mathrm{C}$. Nitric oxide synthase derangements and hypertension in kidney disease. Curr Opin Nephrol Hypertens 2012; 21: 1-6, doi: 10.1097/MNH.0b013e32834d54ca.

54. Verma A, Hirsch DJ, Glatt CE, Ronnett GV, Snyder SH. Carbon monoxide: a putative neural messenger. Science 1993; 259: 381-384, doi: 10.1126/science.7678352.

55. Reis WL, Biancardi VC, Son S, Antunes-Rodrigues J, Stern JE. Enhanced expression of heme oxygenase- 1 and carbon monoxide excitatory effects in oxytocin and vasopressin neurones during water deprivation. J Neuroendocrinol 2012; 24: 653-663, doi: 10.1111/j.1365-2826.2011.02249.x. 
56. Gomes DA, Giusti-Paiva A, Ventura RR, Elias LL, Cunha $F Q$, Antunes-Rodrigues J. Carbon monoxide and nitric oxide modulate hyperosmolality-induced oxytocin secretion by the hypothalamus in vitro. Biosci Rep 2010; 30: 351-357.

57. Mancuso C, Navarra P, Preziosi P. Roles of nitric oxide, carbon monoxide, and hydrogen sulfide in the regulation of the hypothalamic-pituitary-adrenal axis. J Neurochem 2010; 113: 563-575, doi: 10.1111/j.1471-4159.2010.06606.x.

58. Turu G, Hunyady L. Signal transduction of the CB1 cannabinoid receptor. J Mol Endocrinol 2010; 44: 75-85, doi: 10.1677/JME-08-0190.

59. Pertwee RG. Inverse agonism and neutral antagonism at cannabinoid CB1 receptors. Life Sci 2005; 76: 1307-1324, doi: 10.1016/j.lfs.2004.10.025.

60. Ruginsk SG, Oliveira FR, Margatho LO, Vivas L, Elias LL, Antunes-Rodrigues J. Glucocorticoid modulation of neuronal activity and hormone secretion induced by blood volume expansion. Exp Neurol 2007; 206: 192-200, doi: 10.1016/ j.expneurol.2007.04.012.

61. Di S, Maxson MM, Franco A, Tasker JG. Glucocorticoids regulate glutamate and GABA synapse-specific retrograde transmission via divergent nongenomic signaling pathways. J Neurosci 2009; 29: 393-401, doi: 10.1523/JNEUROSCI. 4546-08.2009.

62. Ruginsk SG, Uchoa ET, Elias LL, Antunes-Rodrigues J. Cannabinoid CB1 receptor mediates glucocorticoid effects on hormone secretion induced by volume and osmotic changes. Clin Exp Pharmacol Physiol 2012; 39: 151-154, doi: 10.1111/j.1440-1681.2011.05658.x.

63. Bourque CW, Oliet SH, Richard D. Osmoreceptors, osmoreception, and osmoregulation. Front Neuroendocrinol 1994; 15: 231-274, doi: 10.1006/frne.1994.1010.

64. Hussy N, Deleuze C, Bres V, Moos FC. New role of taurine as an osmomediator between glial cells and neurons in the rat supraoptic nucleus. Adv Exp Med Biol 2000; 483: 227237, doi: 10.1007/0-306-46838-7_25.

65. Jiang S, Yuan H, Duan L, Cao R, Gao B, Xiong YF, et al. Glutamate release through connexin 43 by cultured astrocytes in a stimulated hypertonicity model. Brain Res 2011; 1392: 8-15, doi: 10.1016/j.brainres.2011.03.056.

66. Olsen M, Sarup A, Larsson OM, Schousboe A. Effect of hyperosmotic conditions on the expression of the betaineGABA-transporter (BGT-1) in cultured mouse astrocytes. Neurochem Res 2005; 30: 855-865, doi: 10.1007/s11064005-6879-3.

67. Yuan H, Gao B, Duan L, Jiang S, Cao R, Xiong YF, et al. Acute hyperosmotic stimulus-induced Fos expression in neurons depends on activation of astrocytes in the supraoptic nucleus of rats. J Neurosci Res 2010; 88: 1364-1373.

68. Boudaba C, Linn DM, Halmos KC, Tasker JG. Increased tonic activation of presynaptic metabotropic glutamate receptors in the rat supraoptic nucleus following chronic dehydration. J Physiol 2003; 551: 815-823, doi: 10.1113/ jphysiol.2003.042739.

69. Oliet SH, Bonfardin VD. Morphological plasticity of the rat supraoptic nucleus - cellular consequences. Eur J Neurosci 2010; 32: 1989-1994, doi: 10.1111/j.1460-9568.2010.07514.x.

70. Watanabe E, Hiyama TY, Shimizu H, Kodama R, Hayashi $\mathrm{N}$, Miyata $\mathrm{S}$, et al. Sodium-level-sensitive sodium channel $\mathrm{Na}(\mathrm{x})$ is expressed in glial laminate processes in the sensory circumventricular organs. Am J Physiol Regul Integr Comp Physiol 2006; 290: R568-R576, doi: 10.1152/ ajpregu.00618.2005.

71. Shimizu H, Watanabe E, Hiyama TY, Nagakura A, Fujikawa $A$, Okado $H$, et al. Glial Nax channels control lactate signaling to neurons for brain $[\mathrm{Na}+]$ sensing. Neuron 2007; 54: 59-72, doi: 10.1016/j.neuron.2007.03.014.

72. Coleman CG, Anrather J, ladecola C, Pickel VM. Angiotensin II type 2 receptors have a major somatodendritic distribution in vasopressin-containing neurons in the mouse hypothalamic paraventricular nucleus. Neuroscience 2009; 163: 129142, doi: 10.1016/j.neuroscience.2009.06.032.

73. Antunes-Rodrigues J, Covian MR. Hypothalamic control of sodium chloride and water intake. Acta Physiol Lat Am 1963; 13: 94-100.

74. Vijande $M$, Costales $M$, Marin B. Sex difference in polyethylenglycol-induced thirst. Experientia 1978; 34: 742-743, doi: 10.1007/BF01947296.

75. Carlberg KA, Fregly MJ, Fahey M. Effects of chronic estrogen treatment on water exchange in rats. $A m \mathrm{~J}$ Physiol 1984; 247: E101-E110.

76. Krause EG, Curtis KS, Davis LM, Stowe JR, Contreras RJ. Estrogen influences stimulated water intake by ovariectomized female rats. Physiol Behav 2003; 79: 267-274, doi: 10.1016/S0031-9384(03)00095-7.

77. Mecawi AS, Lepletier A, Araujo IG, Olivares EL, Reis LC. Assessment of brain AT1-receptor on the nocturnal basal and angiotensin-induced thirst and sodium appetite in ovariectomised rats. J Renin Angiotensin Aldosterone Syst 2007; 8: 169-175, doi: 10.3317/jraas.2007.032.

78. Dean SA, Tan J, White R, O'Brien ER, Leenen FH. Regulation of components of the brain and cardiac renin-angiotensin systems by 17 beta-estradiol after myocardial infarction in female rats. Am J Physiol Regul Integr Comp Physiol 2006; 291: R155-R162, doi: 10.1152/ajpregu.00497.2005.

79. Mecawi AS, Lepletier A, Araujo IG, Fonseca FV, Reis LC. Oestrogenic influence on brain AT1 receptor signalling on the thirst and sodium appetite in osmotically stimulated and sodium-depleted female rats. Exp Physiol 2008; 93: 10021010, doi: 10.1113/expphysiol.2008.042713.

80. Robichaud M, Debonnel G. Oestrogen and testosterone modulate the firing activity of dorsal raphe nucleus serotonergic neurones in both male and female rats. J Neuroendocrinol 2005; 17: 179-185, doi: 10.1111/j.1365-2826.2005.01292.x.

81. Sumner BE, Grant KE, Rosie R, Hegele-Hartung C, Fritzemeier $\mathrm{KH}$, Fink $\mathrm{G}$. Effects of tamoxifen on serotonin transporter and 5-hydroxytryptamine(2A) receptor binding sites and mRNA levels in the brain of ovariectomized rats with or without acute estradiol replacement. Brain Res Mol Brain Res 1999; 73: 119-128, doi: 10.1016/S0169-328X(99)00243-0.

82. Donner N, Handa RJ. Estrogen receptor beta regulates the expression of tryptophan-hydroxylase 2 mRNA within serotonergic neurons of the rat dorsal raphe nuclei. Neuroscience 2009; 163: 705-718, doi: 10.1016/j.neuroscience.2009.06.046.

83. Dalmasso C, Amigone JL, Vivas L. Serotonergic system involvement in the inhibitory action of estrogen on induced sodium appetite in female rats. Physiol Behav 2011; 104: 398-407, doi: 10.1016/j.physbeh.2011.04.029.

84. Peysner K, Forsling ML. Effect of ovariectomy and treatment with ovarian steroids on vasopressin release and fluid 
balance in the rat. $J$ Endocrinol 1990; 124: 277-284, doi: 10.1677/joe.0.1240277.

85. Windle RJ, Forsling ML. Variations in oxytocin secretion during the 4-day oestrous cycle of the rat. $J$ Endocrinol 1993; 136: 305-311, doi: 10.1677/joe.0.1360305.

86. Belo NO, Silva-Barra J, Carnio EC, Antunes-Rodrigues J, Gutkowska J, Dos Reis AM. Involvement of atrial natriuretic peptide in blood pressure reduction induced by estradiol in spontaneously hypertensive rats. Regul Pept 2004; 117: 5360, doi: 10.1016/j.regpep.2003.10.007.

87. Vilhena-Franco T, Mecawi AS, Elias LL, Antunes-Rodrigues J. Oestradiol potentiates hormone secretion and neuronal activation in response to hypertonic extracellular volume expansion in ovariectomised rats. J Neuroendocrinol 2011; 23: 481-489, doi: 10.1111/j.1365-2826.2011.02133.x.

88. Mecawi AS, Vilhena-Franco T, Araujo IG, Reis LC, Elias LL,
Antunes-Rodrigues J. Estradiol potentiates hypothalamic vasopressin and oxytocin neuron activation and hormonal secretion induced by hypovolemic shock. Am J Physiol Regul Integr Comp Physiol 2011; 301: R905-R915, doi: 10.1152/ajpregu.00800.2010.

89. Hrabovszky E, Kallo I, Steinhauser A, Merchenthaler I, Coen CW, Petersen SL, et al. Estrogen receptor-beta in oxytocin and vasopressin neurons of the rat and human hypothalamus: Immunocytochemical and in situ hybridization studies. J Comp Neurol 2004; 473: 315-333, doi: 10.1002/cne.20127.

90. Somponpun SJ, Johnson AK, Beltz T, Sladek CD. Estrogen receptor-alpha expression in osmosensitive elements of the lamina terminalis: regulation by hypertonicity. Am J Physiol Regul Integr Comp Physiol 2004; 287: R661-R669, doi: 10.1152/ajpregu.00136.2004. 